\title{
Sinosporangium album gen. nov., sp. nov., a new member of the suborder Streptosporangineae
}

\author{
Yu-Qin Zhang, ${ }^{1} \dagger$ Hong-Yu Liu, ${ }^{1} \dagger \mathrm{Li}-Y a n$ Yu, ${ }^{1}$ Jae-Chan Lee, ${ }^{2}$ \\ Dong-Jin Park, ${ }^{2}$ Chang-Jin Kim, ${ }^{2}$ Li-Hua Xu, ${ }^{3}$ Cheng-Lin Jiang ${ }^{2}$ \\ and Wen-Jun $\mathrm{Li}^{2}$ \\ ${ }^{1}$ Institute of Medicinal Biotechnology, Chinese Academy of Medical Sciences and Peking Union \\ Medical College, Beijing 100050, PR China \\ ${ }^{2}$ Biological Resource Center, Korea Institute of Bioscience and Biotechnology (KRIBB), Daejeon \\ 305-806, Republic of Korea \\ ${ }^{3}$ The Key Laboratory for Microbial Resources of the Ministry of Education, PR China, and \\ Laboratory for Conservation and Utilization of Bio-Resources, Yunnan Institute of Microbiology, \\ Yunnan University, Kunming 650091, PR China
}

Correspondence

Li-Yan Yu yuliyan_2000@yahoo.com

Wen-Jun Li

wjli@ynu.edu.cn
The suborder Streptosporangineae was proposed by Stackebrandt et al. (1997) based on phylogenetic and signature nucleotide analysis. At the time of writing, there were only three families in this suborder, namely, Streptosporangiaceae, Nocardiopsaceae and Thermomonosporaceae, with Streptosporangiaceae as the type family. In this paper, characterization of strain $6014^{\mathrm{T}}$ is reported, with proposals for Sinosporangium gen. nov. and Sinosporangium album sp. nov. in the suborder Streptosporangineae (Stackebrandt et al., 1997).

Strain $6014^{\mathrm{T}}$ was isolated on Czapek agar (Waksman, 1961) incubated at $28{ }^{\circ} \mathrm{C}$ for 3 weeks. The purified strain was

†These authors contributed equally to this work.

The GenBank/EMBL/DDBJ accession number for the 16S rRNA gene sequence of strain $6014^{\top}$ is EU438912.

A supplementary figure and a supplementary table are available with the online version of this paper. maintained on ISP 4 agar and R2A slants at $4{ }^{\circ} \mathrm{C}$ and as glycerol suspensions $(20 \%, \mathrm{v} / \mathrm{v})$ at $-20{ }^{\circ} \mathrm{C}$. Biomass for molecular systematic and chemotaxonomic studies was obtained by cultivation in shake flasks using tryptic soy broth (Difco) at $28{ }^{\circ} \mathrm{C}$ for 10 days. Cultural characteristics of the isolate were determined after growth for $7-28$ days at $28{ }^{\circ} \mathrm{C}$ on R2A, ISP 2, ISP 3, ISP 4 and ISP 5 (Shirling \& Gottlieb, 1966), Czapek agar, nutrient agar (Difco) and potato agar (Waksman, 1961) media. The coverslip technique (Zhou et al., 1998) was employed to observe characteristics of the hyphae, sporangia and spores. The sporangia and spore chain morphologies were recorded by examining gold-coated dehydrated specimens of 14 day cultures from R2A and ISP 4 agar by scanning electron microscopy (Quanta; FEI).

Growth was tested at $0,4,10,15,20,28-37$ (at intervals of $\left.1{ }^{\circ} \mathrm{C}\right), 40,45$ and $55^{\circ} \mathrm{C}$ on R2A. Other physiological and biochemical tests were performed at $28{ }^{\circ} \mathrm{C}$. The $\mathrm{pH}$ range was examined at $\mathrm{pH} 4.0-11.0$ (at intervals of $0.5 \mathrm{pH}$ units). 
Tolerance to sodium chloride $[0,1,3$ and $5-10 \%(\mathrm{w} / \mathrm{v})$, at intervals of $0.5 \%$ ] was examined using ISP 4 as basal medium. Carbon source utilization was tested as described by Shirling \& Gottlieb (1966) and also using Biolog GEN III (MicroPlate) according to the manufacturer's instructions. Qualitative enzyme tests and acid production from carbohydrates were determined by using the API ZYM and API $50 \mathrm{CH}$ systems (bioMérieux) according to the manufacturer's instructions. Other physiological tests and antimicrobial activities of the strain were examined according to previously described procedures (Yuan et al., 2008).

Strain $6014^{\mathrm{T}}$ grew well on R2A, ISP 4, ISP 5, Czapek solution agar, nutrient agar and potato agar media at 28$32{ }^{\circ} \mathrm{C}$ and $\mathrm{pH}$ 7.0-7.5. White to buff branching vegetative hyphae developed well and abundant white aerial hyphae were produced on the above media, whereas the strain grew very slowly with few aerial hyphae on ISP 2 and ISP 3 media. Diffusible pigments were not observed on any test media. Globose sporangia formed singly from the aerial hyphae (Fig. 1a). The sporangia were a mean size of 2.8 $3.0 \times 3.4-4.2 \mu \mathrm{m}$ (Fig. 1b) and contained coiled spore chains (Fig. 1a). The non-motile, smooth-surfaced, cylindrical spores were about $0.5-0.6 \times 0.6-1.2 \mu \mathrm{m}$. Gelatin, starch, urea and aesculin were not hydrolysed. Milk was not coagulated or peptonized and $\mathrm{H}_{2} \mathrm{~S}$ was not produced. The isolate could use most of the carbon sources listed in Biolog GEN III as sole carbon sources (Supplementary Table S1, available in IJSEM Online) for energy and growth except $\alpha$-lactose, D-galactose and myo-inositol. Detailed physiological and biochemical characteristics of strain $6014^{\mathrm{T}}$ are given in the species description.

The whole-cell sugar pattern and diagnostic diaminopimelic acid isomers were determined by TLC (Lechevalier \& Lechevalier, 1965, 1980). Polar lipids were extracted and examined by two-dimensional TLC and identified using procedures described by Minnikin et al. (1984). Menaquinones were isolated using the method of Collins et al. (1977) and analysed by HPLC (Groth et al., 1997). Analysis of the whole-cell fatty acid pattern followed described methods using the MIDI system (Microbial ID) (Kroppenstedt, 1985; Meier et al., 1993). Extraction of genomic DNA and PCR amplification of the 16S rRNA gene were done as described by Li et al. (2007). The DNA $\mathrm{G}+\mathrm{C}$ content was determined by reverse-phase HPLC of nucleosides according to Mesbah et al. (1989). 16S rRNA gene multiple alignments with sequences of most closely related taxa and calculations of sequence similarity levels were carried out using CLUSTAL X (Thompson et al., 1997). A phylogenetic tree and distance matrix were reconstructed using the neighbour-joining method of Saitou \& Nei (1987) from $K_{\text {nuc }}$ values (Kimura, 1980, 1983) using MEGA version 4.0 (Tamura et al., 2007). A maximum-likelihood (Felsenstein, 1981) tree (not shown) was generated using the treeing algorithm contained in the PHYLIP package (Felsenstein, 1993). Topology of the phylogenetic tree was evaluated by the bootstrap resampling method of Felsenstein (1985) with 1000 replicates.
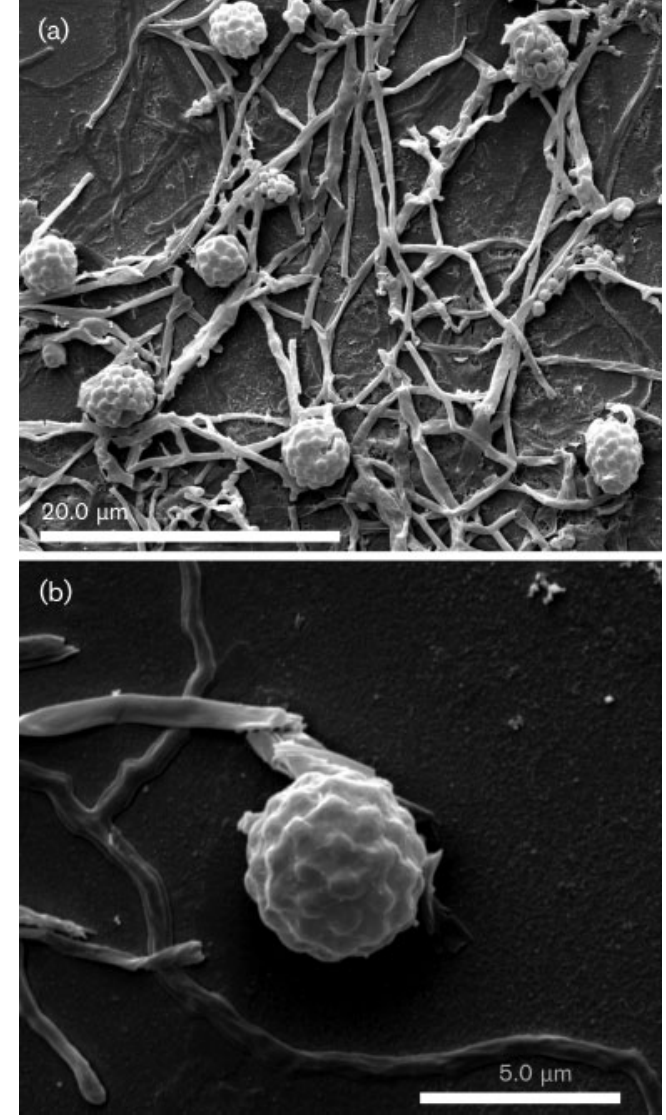

Fig. 1. Scanning electron micrographs of strain $6014^{\top}$ after growth for 14 days at $28{ }^{\circ} \mathrm{C}$ on ISP 4 agar showing spore chains (a; bar, $20.0 \mu \mathrm{m}$ ) and a sporangium (b; bar, $5.0 \mu \mathrm{m}$ ).

Whole-cell hydrolysates of strain $6014^{\mathrm{T}}$ contained ribose, galactose and arabinose. The diagnostic diamino acid was meso-diaminopimelic acid. The polar lipids comprised phosphatidylmethylethanolamine, phosphatidylethanolamine, hydroxy-phosphatidylethanolamine, $\mathrm{N}$-acetylglucosamine-containing phospholipids, two unknown phospholipids and an unknown glycolipid (Supplementary Fig. S1). The menaquinone system contained MK-9 $\left(\mathrm{H}_{2}\right)$ (53.1\%) and MK-9 $\left(\mathrm{H}_{4}\right)(46.9 \%)$. The major fatty acids detected were saturated, iso- and 10-methyl branched fatty acids. The detailed cellular fatty acid profile is as follows: $\mathrm{C}_{12: 0}$ $(1.0 \%), \mathrm{C}_{13: 0}(0.6 \%), \mathrm{C}_{14: 0}(10.4 \%), \mathrm{C}_{15: 0}(2.1 \%), \mathrm{C}_{16: 0}$ $(20.9 \%), \mathrm{C}_{17: 0}(0.6 \%), \mathrm{C}_{18: 0}(1.0 \%)$, iso- $\mathrm{C}_{14: 0}(1.7 \%)$, iso- $\mathrm{C}_{15: 0}(10.2 \%)$, iso- $\mathrm{C}_{16: 0}(8.3 \%)$, iso- $\mathrm{C}_{17: 0}(2.0 \%)$, anteiso- $\mathrm{C}_{17: 0}(0.7 \%), \quad 10$-methyl- $\mathrm{C}_{16: 0} \quad(10.1 \%), \quad 10-$

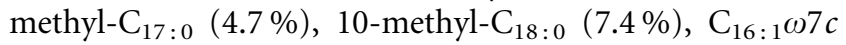
$(8.1 \%), \mathrm{C}_{17: 1} \omega 8 c(2.2 \%), \mathrm{C}_{18: 1} \omega 9 c(5.9 \%)$. The genomic DNA G + C content was $69.4 \mathrm{~mol} \%$.

BLAST search results using the $16 \mathrm{~S}$ rRNA gene sequence of strain $6014^{\mathrm{T}}$ showed that the novel isolate exhibited highest similarities with members of the suborder Streptosporangineae, such as Streptosporangium violaceochromogenes DSM $43849^{\mathrm{T}}(96.1 \%)$. However, in the phylogenetic tree 


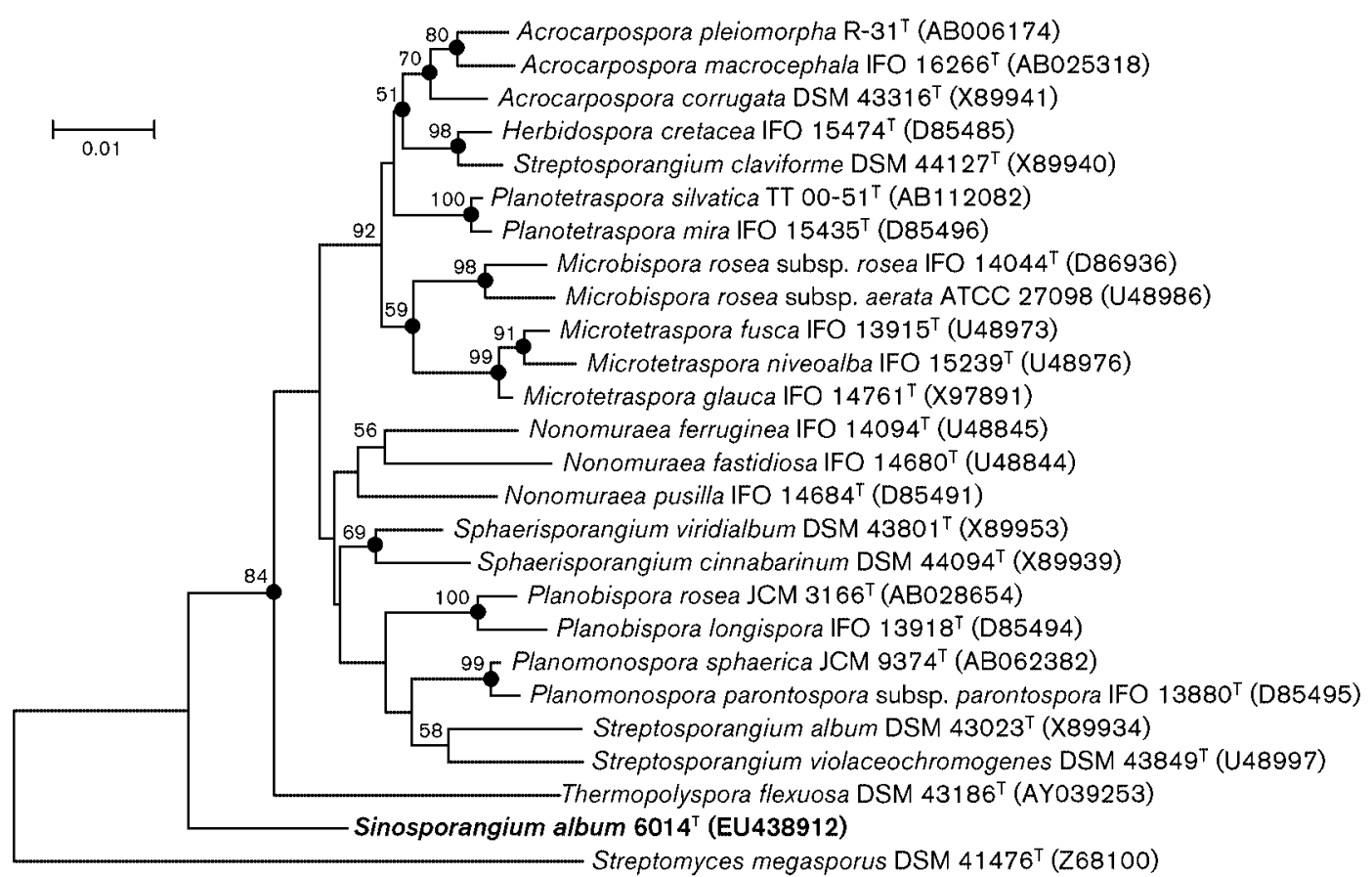

Fig. 2. Neighbour-joining phylogenetic tree, based on $16 \mathrm{~S}$ rRNA gene sequences, showing the position of strain $6014^{\top}$ among its phylogenetically nearest neighbours. Bootstrap percentages (based on 1000 replications) $>50 \%$ are shown at branch points. Filled circles indicate that the corresponding nodes were also recovered in the tree generated with the maximumlikelihood method. Bar, 0.01 substitutions per nucleotide position.

Table 1. Signature nucleotide patterns of the genus Sinosporangium and related genera

Genera: 1, Sinosporangium gen. nov.; 2, Sphaerosporangium; 3, Streptosporangium; 4, Planotetraspora; 5, Planomonospora; 6, Planobispora; 7, Nonomuraea; 8, Microtetraspora; 9, Microbispora; 10, Herbidospora; 11, Acrocarpospora.

\begin{tabular}{|c|c|c|c|c|c|c|c|c|c|c|c|}
\hline Position & 1 & 2 & 3 & 4 & 5 & 6 & 7 & 8 & 9 & 10 & 11 \\
\hline 263 & G & G & A & G & G & G & G & G & A & G & G \\
\hline 264 & U & U & $\mathrm{C}$ & U & U & U & U & $\mathrm{U}$ & $\mathrm{U}$ & $\mathrm{U}$ & $\mathrm{U}$ \\
\hline $442: 492$ & $\mathrm{G}-\mathrm{C}$ & $\mathrm{G}-\mathrm{C}$ & $\mathrm{G}-\mathrm{C}$ & G-U & $\mathrm{G}-\mathrm{C}$ & $\mathrm{G}-\mathrm{C}$ & $\mathrm{G}-\mathrm{C}$ & $\mathrm{G}-\mathrm{U}$ & $\mathrm{G}-\mathrm{U}$ & $\mathrm{G}-\mathrm{U}$ & $\mathrm{G}-\mathrm{U}$ \\
\hline $594: 645$ & $\mathrm{C}-\mathrm{G}$ & $\mathrm{C}-\mathrm{G}$ & $\mathrm{C}-\mathrm{G}$ & U-G & $\mathrm{C}-\mathrm{G}$ & $\mathrm{C}-\mathrm{G}$ & $\mathrm{C}-\mathrm{G}$ & $\mathrm{C}-\mathrm{G}$ & $\mathrm{C}-\mathrm{G} / \mathrm{U}-\mathrm{G}$ & $\mathrm{C}-\mathrm{G}$ & $\mathrm{C}-\mathrm{G}$ \\
\hline 595 & G & G & A & G & G & G & G & G & G & G & G \\
\hline $600: 638$ & $\mathrm{C}-\mathrm{G}$ & $\mathrm{U}-\mathrm{G}$ & $\mathrm{G}-\mathrm{C}$ & U-G & $\mathrm{G}-\mathrm{C}$ & $\mathrm{G}-\mathrm{C}$ & $\mathrm{U}-\mathrm{G}$ & $\mathrm{U}-\mathrm{G}$ & U-G & U-G & U-G \\
\hline $602: 636$ & $\mathrm{C}-\mathrm{G}$ & $\mathrm{C}-\mathrm{G}$ & $\mathrm{G}-\mathrm{U}$ & $\mathrm{C}-\mathrm{G}$ & $\mathrm{A}-\mathrm{U}$ & $\mathrm{A}-\mathrm{U}$ & $\mathrm{C}-\mathrm{G}$ & $\mathrm{C}-\mathrm{G}$ & $\mathrm{C}-\mathrm{G}$ & $\mathrm{C}-\mathrm{G}$ & $\mathrm{C}-\mathrm{G}$ \\
\hline $603: 635$ & $\mathrm{C}-\mathrm{G}$ & $\mathrm{C}-\mathrm{G}$ & $\mathrm{U}-\mathrm{A}$ & $\mathrm{C}-\mathrm{G}$ & $\mathrm{U}-\mathrm{A}$ & $\mathrm{U}-\mathrm{A}$ & $\mathrm{C}-\mathrm{C}$ & $\mathrm{C}-\mathrm{C}$ & $\mathrm{C}-\mathrm{C}$ & $\mathrm{C}-\mathrm{C}$ & $\mathrm{C}-\mathrm{C}$ \\
\hline 625 & $\mathrm{C}$ & G & $\mathrm{C}$ & G & $\mathrm{C}$ & G & G & $\mathrm{C}$ & A & A & G \\
\hline 626 & $\mathrm{C}$ & $\mathrm{U}$ & $\mathrm{C}$ & $\mathrm{C}$ & $\mathrm{C}$ & $\mathrm{C}$ & C & $\mathrm{U}$ & $\mathrm{C}$ & $\mathrm{U}$ & C \\
\hline 627 & G & G & A & G & A & G & G & A & G & G & G \\
\hline $657: 749$ & $\mathrm{G}-\mathrm{C}$ & $\mathrm{G}-\mathrm{C}$ & $\mathrm{G}-\mathrm{C}$ & $\mathrm{G}-\mathrm{U}$ & $\mathrm{C}-\mathrm{C}$ & $\mathrm{G}-\mathrm{C}$ & $\mathrm{G}-\mathrm{C}$ & $\mathrm{G}-\mathrm{U}$ & $\mathrm{G}-\mathrm{U}$ & $\mathrm{G}-\mathrm{U}$ & $\mathrm{G}-\mathrm{U}$ \\
\hline $658: 748$ & $\mathrm{C}-\mathrm{G}$ & $\mathrm{U}-\mathrm{A}$ & $\mathrm{U}-\mathrm{A}$ & $\mathrm{C}-\mathrm{U}$ & $\mathrm{U}-\mathrm{A}$ & $\mathrm{U}-\mathrm{A}$ & $\mathrm{U}-\mathrm{A}$ & $\mathrm{C}-\mathrm{U}$ & $\mathrm{C}-\mathrm{U}$ & $\mathrm{C}-\mathrm{U}$ & $\mathrm{C}-\mathrm{U}$ \\
\hline $659: 746$ & $\mathrm{~A}-\mathrm{U}$ & $\mathrm{A}-\mathrm{U}$ & $\mathrm{A}-\mathrm{U}$ & $\mathrm{U}-\mathrm{A}$ & $\mathrm{A}-\mathrm{U}$ & $\mathrm{A}-\mathrm{U}$ & $\mathrm{A}-\mathrm{U}$ & $\mathrm{U}-\mathrm{A}$ & U-A & $\mathrm{U}-\mathrm{A}$ & $\mathrm{U}-\mathrm{G}$ \\
\hline $668: 738$ & $\mathrm{~A}-\mathrm{U}$ & $C-G$ & $\mathrm{~A}-\mathrm{U}$ & $\mathrm{C}-\mathrm{G}$ & $C-G$ & $C-G$ & $C-G$ & $C-G$ & $\mathrm{C}-\mathrm{G}$ & $\mathrm{C}-\mathrm{G}$ & $\mathrm{C}-\mathrm{G}$ \\
\hline $669: 737$ & $\mathrm{G}-\mathrm{C}$ & $\mathrm{A}-\mathrm{U}$ & $\mathrm{G}-\mathrm{C}$ & $\mathrm{A}-\mathrm{U}$ & $\mathrm{A}-\mathrm{U}$ & $\mathrm{A}-\mathrm{U}$ & $\mathrm{A}-\mathrm{U}$ & $\mathrm{A}-\mathrm{U}$ & $\mathrm{A}-\mathrm{U}$ & $\mathrm{A}-\mathrm{U}$ & $\mathrm{A}-\mathrm{U}$ \\
\hline $671: 735$ & $\mathrm{~A}-\mathrm{U}$ & $\mathrm{G}-\mathrm{C}$ & $\mathrm{A}-\mathrm{U}$ & $\mathrm{G}-\mathrm{C}$ & $\mathrm{G}-\mathrm{C}$ & $\mathrm{G}-\mathrm{C}$ & $\mathrm{G}-\mathrm{C}$ & $\mathrm{G}-\mathrm{C}$ & $\mathrm{G}-\mathrm{C}$ & $\mathrm{G}-\mathrm{C}$ & $\mathrm{G}-\mathrm{C}$ \\
\hline $835: 851$ & $\mathrm{G}-\mathrm{C}$ & $\mathrm{G}-\mathrm{U}$ & $\mathrm{G}-\mathrm{U}$ & $\mathrm{G}-\mathrm{C}$ & $\mathrm{G}-\mathrm{U}$ & $\mathrm{G}-\mathrm{C}$ & $\mathrm{G}-\mathrm{C}$ & $\mathrm{G}-\mathrm{C}$ & $\mathrm{G}-\mathrm{C}$ & G-C & $\mathrm{G}-\mathrm{C}$ \\
\hline $990: 1215$ & $\mathrm{U}-\mathrm{G}$ & $\mathrm{C}-\mathrm{G}$ & $\mathrm{C}-\mathrm{G}$ & $\mathrm{G}-\mathrm{C} / \mathrm{G}-\mathrm{U}$ & $\mathrm{G}-\mathrm{U}$ & $\mathrm{G}-\mathrm{C}$ & $\mathrm{G}-\mathrm{U}$ & $\mathrm{G}-\mathrm{C}$ & $\mathrm{G}-\mathrm{C}$ & $\mathrm{G}-\mathrm{U}$ & $\mathrm{G}-\mathrm{C}$ \\
\hline $1012: 1017$ & $\mathrm{G}-\mathrm{U}$ & $\mathrm{G}-\mathrm{C}$ & $\mathrm{A}-\mathrm{U}$ & $\mathrm{A}-\mathrm{U}$ & $\mathrm{A}-\mathrm{U}$ & $\mathrm{A}-\mathrm{U}$ & $\mathrm{A}-\mathrm{U}$ & $\mathrm{A}-\mathrm{U}$ & $\mathrm{A}-\mathrm{U}$ & $\mathrm{A}-\mathrm{U}$ & $\mathrm{A}-\mathrm{U}$ \\
\hline 1116 & G & $\mathrm{U}$ & $\mathrm{U}$ & $\mathrm{U}$ & $\mathrm{U}$ & $\mathrm{U}$ & $\mathrm{U}$ & $\mathrm{U}$ & $\mathrm{U}$ & $\mathrm{U}$ & $\mathrm{U}$ \\
\hline 1137 & $\mathrm{C}$ & $\mathrm{U}$ & $\mathrm{U}$ & $\mathrm{U}$ & U & U & U & $\mathrm{U}$ & $\mathrm{U}$ & $\mathrm{U}$ & $\mathrm{U}$ \\
\hline $1263: 1272$ & $\mathrm{G}-\mathrm{C}$ & $\mathrm{G}-\mathrm{U}$ & $\mathrm{A}-\mathrm{U}$ & $\mathrm{A}-\mathrm{U}$ & $\mathrm{A}-\mathrm{U}$ & $\mathrm{A}-\mathrm{U}$ & $\mathrm{A}-\mathrm{U}$ & $\mathrm{A}-\mathrm{U}$ & $\mathrm{A}-\mathrm{U}$ & $\mathrm{A}-\mathrm{U}$ & $\mathrm{G}-\mathrm{U}$ \\
\hline $1436: 1465$ & $\mathrm{C}-\mathrm{U}$ & $\mathrm{G}-\mathrm{C}$ & $\mathrm{G}-\mathrm{C}$ & $\mathrm{G}-\mathrm{C}$ & $\mathrm{G}-\mathrm{C}$ & $\mathrm{G}-\mathrm{C}$ & $\mathrm{G}-\mathrm{C}$ & $\mathrm{G}-\mathrm{C}$ & $\mathrm{G}-\mathrm{C}$ & $\mathrm{G}-\mathrm{C}$ & $\mathrm{G}-\mathrm{C}$ \\
\hline
\end{tabular}


Table 2. Morphological and chemotaxonomic characteristics of strain $6014^{\top}$ and members of genera in the family Streptosporangiaceae

Taxa: 1, 6014 $;$ 2, Streptosporangium; 3, Acrocarpospora; 4, Herbidospora; 5, Microbispora; 6, Microtetraspora; 7, Nonomuraea; 8, Planobispora; 9, Planomonospora; 10, Planotetraspora; 11, Sphaerisporangium; 12, Thermopolyspora. Data were taken from this and previous studies (Greiner-Mai et al., 1987; Goodfellow et al., 1990; Kudo et al., 1993; Tamura et al., 2000; Stackebrandt et al., 2001; Tamura \& Sakane, 2004; Ara \& Kudo, 2007; Goodfellow et al., 2005; Cao et al., 2009). For all taxa, the fatty acid type [saturated fatty acids, unsaturated fatty acids, iso-fatty acids, variable and methyl-branched fatty acids (Kroppenstedt, 1985)] was 3c. +, Present; -, absent.

\begin{tabular}{|c|c|c|c|c|c|c|c|c|c|c|c|c|}
\hline Characteristic & 1 & 2 & 3 & 4 & 5 & 6 & 7 & 8 & 9 & 10 & 11 & 12 \\
\hline $\begin{array}{l}\text { Sporangium } \\
\text { formation }\end{array}$ & $\begin{array}{l}\text { Globose } \\
\text { sporangia } \\
\text { on aerial } \\
\text { hyphae }\end{array}$ & $\begin{array}{l}\text { Globose } \\
\text { sporangia } \\
\text { on aerial } \\
\text { hyphae }\end{array}$ & $\begin{array}{c}\text { Club or } \\
\text { globose } \\
\text { spore vesicles } \\
\text { on aerial } \\
\text { hyphae }\end{array}$ & $\begin{array}{c}\text { Straight } \\
\text { chains of smooth- } \\
\text { surfaced spores on } \\
\text { aerial hyphae }\end{array}$ & $\begin{array}{l}\text { Smooth-surfaced } \\
\text { spores in } \\
\text { characteristic } \\
\text { longitudinal pairs } \\
\text { on aerial hyphae }\end{array}$ & $\begin{array}{l}\text { Spore chains } \\
\text { typically } \\
\text { containing four } \\
\text { smooth-surfaced } \\
\text { spores on short } \\
\text { aerial hyphae }\end{array}$ & $\begin{array}{c}\text { Spore } \\
\text { chains or } \\
\text { pseudo- } \\
\text { sporangia formed } \\
\text { on aerial } \\
\text { hyphae }\end{array}$ & $\begin{array}{c}\text { Cylindrical } \\
\text { to clavate spore } \\
\text { vesicles } \\
\text { containing } \\
\text { longitudinal pairs } \\
\text { of spores on aerial } \\
\text { hyphae }\end{array}$ & $\begin{array}{l}\text { Cylindrical } \\
\text { to clavate } \\
\text { spore vesicles } \\
\text { containing } \\
\text { single spores } \\
\text { on aerial } \\
\text { hyphae }\end{array}$ & $\begin{array}{l}\text { Spore vesicles } \\
\text { containing } \\
\text { four spores } \\
\text { on aerial } \\
\text { hyphae }\end{array}$ & $\begin{array}{l}\text { Globose } \\
\text { sporangia } \\
\text { on aerial } \\
\text { hyphae }\end{array}$ & $\begin{array}{c}\text { Hooked or } \\
\text { irregular spiral } \\
\text { chains of four to } \\
\text { ten warty to spiny } \\
\text { ornamented } \\
\text { spores on aerial } \\
\text { hyphae }\end{array}$ \\
\hline Motile spores & - & - & - & - & - & - & - & + & + & + & - & - \\
\hline $\begin{array}{l}\text { Whole-cell sugar } \\
\text { pattern }^{\star}\end{array}$ & A & B & B, C & B & B, C & B, C & B, C & B & B & A, D & B & C \\
\hline $\begin{array}{l}\text { Major } \\
\text { menaquinone(s) }\end{array}$ & $\begin{array}{c}\text { MK- } 9\left(\mathrm{H}_{2}\right. \\
\left.\mathrm{H}_{4}\right)\end{array}$ & $\begin{array}{c}\text { MK- } 9\left(\mathrm{H}_{2},\right. \\
\left.\mathrm{H}_{4}\right)\end{array}$ & MK-9( $\left.\mathrm{H}_{2}, \mathrm{H}_{4}, \mathrm{H}_{6}\right)$ & MK-10 $\left(\mathrm{H}_{4}, \mathrm{H}_{6}, \mathrm{H}_{8}\right)$ & MK- $9\left(\mathrm{H}_{2}, \mathrm{H}_{4}\right)$ & MK-9 $\left(\mathrm{H}_{2}, \mathrm{H}_{4}\right)$ & MK-9 $\left(\mathrm{H}_{2}, \mathrm{H}_{4}\right)$ & MK-9 $\left(\mathrm{H}_{2}, \mathrm{H}_{4}\right)$ & MK-9 $\left(\mathrm{H}_{2}\right)$ & MK-9 $\left(\mathrm{H}_{4}\right)$ & MK-9 $\left(\mathrm{H}_{4}\right)$ & MK-9 $\left(\mathrm{H}_{2}, \mathrm{H}_{4}\right)$ \\
\hline $\begin{array}{l}\text { Phospholipid } \\
\text { type } \dagger\end{array}$ & PIV & PIV & PIV, PII & PIV & PIV & PIV & PIV & PIV & PIV & PIV & PIV & PIV \\
\hline $\begin{array}{l}\text { DNA G + C } \\
\text { content }(\mathrm{mol} \%)\end{array}$ & 69.4 & $69-71$ & $68-69$ & $69-71$ & $71-73$ & 69-71 & $64-69$ & $70-71$ & 72 & 71 & $67-72$ & 77 \\
\hline
\end{tabular}

*Whole-cell sugar patterns of actinomycetes containing meso-diaminopimelic acid: A, arabinose and galactose; B, madurose; C, no diagnostic sugar; D, arabinose and xylose (Lechevalier \& Lechevalier, 1970).

$†$ Diagnostic phospholipids: PII, phosphatidylethanolamine; PIV, glucosamine (with phosphatidylethanolamine and phosphatidylmethylethanolamine variable) (Lechevalier et al., 1977). 
(not shown) based on the 16S rRNA gene sequences of all genera in the suborder Streptosporangineae, strain $6014^{\mathrm{T}}$ formed a distinct lineage among families in the suborder Streptosporangineae, next to the family Streptosporangiaceae (Fig. 2), which showed that strain $6014^{\mathrm{T}}$ could not be placed into any known family. Analysis of $16 \mathrm{~S}$ rRNA signature nucleotide patterns demonstrated that strain $6014^{\mathrm{T}}$ contained the signature nucleotide pattern defined for the suborder Streptosporangineae, namely $127: 234$ (AU), 657:749 (G-C) and 955: $1225(\mathrm{C}-\mathrm{G})$ (Stackebrandt et al., 1997); additionally, strain $6014^{\mathrm{T}}$ possessed its own signature nucleotides, which distinguished it from its nearest neighbour, namely 1116 (G), 1137 (C) and 1436:1465 (C-U), instead of $1116(\mathrm{U}), 1137(\mathrm{U})$ and $1436: 1465$ (G-C) for the family Streptosporangiaceae. The specific diagnostic nucleotide signature patterns of the isolate and genera in the family Streptosporangiaceae are listed in Table 1.

Based on its phylogenetic position (Fig. 2) and chemotaxonomic data (Table 2), it is proposed that strain $6014^{\mathrm{T}}$ represents a novel species in a new genus, Sinosporangium album gen. nov., sp. nov.

\section{Description of Sinosporangium gen. nov.}

Sinosporangium [Si.no.spo.ran'gi.um. M.L. n. sina China; N.L. n. sporangium from Gr. n. spora a seed and, in biology, a spore and Gr. n. angeion (Latin transliteration angium) vessel, sporangium; N.L. neut. n. Sinosporangium an organism isolated in China bearing sporangia].

Cells are Gram-positive and form branching hyphae. Globose sporangia are borne on aerial mycelia. Coiled spore chains are contained in the sporangia. Smoothsurfaced spores are non-motile. Grows at $\mathrm{pH} 6.5-8.5$ and 10-37 ${ }^{\circ} \mathrm{C}$. Catalase-positive, oxidase-negative. The diagnostic amino acid of the peptidoglycan is meso-diaminopimelic acid. Whole-cell hydrolysates contain arabinose, galactose and ribose. Phospholipids consist of phosphatidylmethylethanolamine, phosphatidylethanolamine, hydroxyphosphatidylethanolamine, $\mathrm{N}$-acetylglucosamine-containing phospholipids, two unknown phospholipids and an unknown glycolipid. The menaquinone system contains MK-9 $\left(\mathrm{H}_{2}\right) \quad(53.1 \%)$ and MK-9 $\left(\mathrm{H}_{4}\right) \quad(46.9 \%)$. Major cellular fatty acids are $\mathrm{C}_{16: 0}, \mathrm{C}_{14: 0}$, iso- $\mathrm{C}_{15: 0}$ and 10methyl- $\mathrm{C}_{16: 0}$. The $16 \mathrm{~S}$ rRNA contains a genus-specific diagnostic nucleotide signature pattern, namely $600: 638$ (C-G), $658: 748$ (C-G), 990:1215 (U-G), 1012:1017 (G$\mathrm{U})$ and $1263: 1272(\mathrm{G}-\mathrm{C})$. The type species is Sinosporangium album.

\section{Description of Sinosporangium album sp. nov.}

Sinosporangium album (al'bum. L. neut. adj. album white).

In addition to properties given for the genus, forms white to buff branching substrate mycelia and white aerial mycelia. No diffusible pigment is produced on any of the media tested. Optimum growth occurs at $\mathrm{pH}$ 7.0-7.5 and $28-32{ }^{\circ} \mathrm{C}$. Can tolerate up to $1 \%(\mathrm{w} / \mathrm{v}) \mathrm{NaCl}$ at $28{ }^{\circ} \mathrm{C}$. Positive for alkaline phosphatase, leucine arylamidase, valine arylamidase, trypsin, acid phosphatase, naphthol-AS-BI-phosphohydrolase, $\alpha$-glucosidase and $\beta$ glucosidase activities. Negative for gelatin liquefaction, milk coagulation and peptonization, hydrolysis of starch, urea and aesculin, nitrate reduction, $\mathrm{H}_{2} \mathrm{~S}$ production and hydrolysis of cellulose. Most of the substrates listed in Biolog GEN III can be utilized as sole carbon sources for energy and growth, except $\alpha$-lactose, D-galactose and myoinositol. Acid can be produced from D-adonitol, D-glucose, trehalose and potassium 5-ketogluconate.

The type strain is $6014^{\mathrm{T}}\left(=\mathrm{DSM} 45181^{\mathrm{T}}=\mathrm{KCTC} 19655^{\mathrm{T}}\right)$, isolated from soil collected in Qinghai, China. The genomic DNA $\mathrm{G}+\mathrm{C}$ content of the type strain is $69.4 \mathrm{~mol} \%$.

\section{Acknowledgements}

This research was supported by National Facilities and Information Infrastructure for Science and Technology (2005DKA21203), National S\&T Major Special Project on Major New Drug Innovation (nos: 2009ZX09301-003 and 2009ZX09302-004), the National Natural Science Foundation of China (NSFC) (nos: 30970008 and 30970038), State-level public welfare scientific research institutes for basic R\&D special fund (no. IMBF200915) and the 21C Frontier Program of Microbial Genomics and Applications from the MEST, Korea.

\section{References}

Ara, I. \& Kudo, T. (2007). Sphaerosporangium gen. nov., a new member of the family Streptosporangiaceae, with descriptions of three new species as Sphaerosporangium melleum sp. nov., Sphaerosporangium rubeum sp. nov. and Sphaerosporangium cinnabarinum sp. nov., and transfer of Streptosporangium viridialbum Nonomura and Ohara 1960 to Sphaerosporangium viridialbum comb. nov. Actinomycetologica 21, 11-21.

Cao, Y.-R., Jiang, Y., Xu, L.-H. \& Jiang, C.-L. (2009). Sphaerisporangium flaviroseum sp. nov. and Sphaerisporangium album sp. nov., isolated from forest soil in China. Int J Syst Evol Microbiol 59, 16791684.

Collins, M. D., Pirouz, T., Goodfellow, M. \& Minnikin, D. E. (1977). Distribution of menaquinones in actinomycetes and corynebacteria. J Gen Microbiol 100, 221-230.

Felsenstein, J. (1981). Evolutionary trees from DNA sequences: a maximum likelihood approach. J Mol Evol 17, 368-376.

Felsenstein, J. (1985). Confidence limits on phylogenies: an approach using the bootstrap. Evolution 39, 783-791.

Felsenstein, J. (1993). PHYLIP (phylogeny inference package), version 3.5c. Distributed by the author. Department of Genome Sciences, University of Washington, Seattle, USA.

Goodfellow, M., Stanton, L. J., Simpson, K. E. \& Minnikin, D. E. (1990). Numerical and chemical classification of Actinoplanes and some related actinomycetes. J Gen Microbiol 136, 19-36.

Goodfellow, M., Maldonado, L. A. \& Quintana, E. T. (2005). Reclassification of Nonomuraea flexuosa (Meyer 1989) Zhang et al. 1998 as Thermopolyspora flexuosa gen. nov., comb. nov., nom. rev. Int J Syst Evol Microbiol 55, 1979-1983. 
Greiner-Mai, E., Kroppenstedt, R. M., Korn-Wendisch, F. \& Kutzner, H. J. (1987). Morphological and biochemical characterization and emended descriptions of thermophilic actinomycetes. Syst Appl Microbiol 9, 97-109.

Groth, I., Schumann, P., Rainey, F. A., Martin, K., Schuetze, B. \& Augsten, K. (1997). Demetria terragena gen. nov., sp. nov., a new genus of actinomycetes isolated from compost soil. Int J Syst Bacteriol 47, 1129-1133.

Kimura, M. (1980). A simple method for estimating evolutionary rates of base substitutions through comparative studies of nucleotide sequences. J Mol Evol 16, 111-120.

Kimura, M. (1983). The Neutral Theory of Molecular Evolution. Cambridge: Cambridge University Press.

Kroppenstedt, R. M. (1985). Fatty acid and menaquinone analysis of actinomycetes and related organisms. In Chemical Methods in Bacterial Systematics (Society for Applied Bacteriology Technical Series vol. 20), pp. 173-199. Edited by M. Goodfellow \& D. E. Minnikin. New York: Academic Press.

Kudo, T., Itoh, T., Miyadoh, S., Shomura, T. \& Seino, A. (1993). Herbidospora gen. nov., a new genus of the family Streptosporangiaceae Goodfellow et al. 1990. Int J Syst Bacteriol 43, 319-328.

Lechevalier, H. \& Lechevalier, M. P. (1965). Classification of aerobic actinomycetes based on their morphology and their chemical composition. Ann Inst Pasteur (Paris) 108, 662-673 (in French).

Lechevalier, M. P. \& Lechevalier, H. A. (1970). Chemical composition as a criterion in the classification of aerobic actinomycetes. Int J Syst Bacteriol 20, 435-443.

Lechevalier, M. P. \& Lechevalier, H. A. (1980). The chemotaxonomy of actinomycetes. In Actinomycete Taxonomy (SIM Special Publication no. 6), pp. 227-291. Edited by A. Dietz \& D. W. Thayer Arlington, VA: Society for Industrial Microbiology.

Lechevalier, M. P., De Bièvre, C. \& Lechevalier, H. A. (1977). Chemotaxonomy of aerobic actinomycetes: phospholipid composition. Biochem Syst Ecol 5, 249-260.

Li, W.-J., Xu, P., Schumann, P., Zhang, Y.-Q., Pukall, R., Xu, L.-H., Stackebrandt, E. \& Jiang, C.-L. (2007). Georgenia ruanii sp. nov., a novel actinobacterium isolated from forest soil in Yunnan (China), and emended description of the genus Georgenia. Int J Syst Evol Microbiol 57, 1424-1428.
Meier, A., Kirschner, P., Schröder, K. H., Wolters, J., Kroppenstedt, R. M. \& Böttger, E. C. (1993). Mycobacterium intermedium sp. nov. Int J Syst Bacteriol 43, 204-209.

Mesbah, M., Premachandran, U. \& Whitman, W. B. (1989). Precise measurement of the $\mathrm{G}+\mathrm{C}$ content of deoxyribonucleic acid by highperformance liquid chromatography. Int J Syst Bacteriol 39, 159-167.

Minnikin, D. E., O’Donnell, A. G., Goodfellow, M., Alderson, G., Athalye, M., Schaal, A. \& Parlett, J. H. (1984). An integrated procedure for the extraction of bacterial isoprenoid quinones and polar lipids. J Microbiol Methods 2, 233-241.

Saitou, N. \& Nei, M. (1987). The neighbor-joining method: a new method for reconstructing phylogenetic trees. Mol Biol Evol 4, 406-425.

Shirling, E. B. \& Gottlieb, D. (1966). Methods for characterization of Streptomyces species. Int J Syst Bacteriol 16, 313-340.

Stackebrandt, E., Rainey, F. A. \& Ward-Rainey, N. L. (1997). Proposal for a new hierarchic classification system, Actinobacteria classis nov. Int J Syst Bacteriol 47, 479-491.

Stackebrandt, E., Wink, J., Steiner, U. \& Kroppenstedt, R. M. (2001) Nonomuraea dietzii sp. nov. Int J Syst Evol Microbiol 51, 1437-1441.

Tamura, T. \& Sakane, T. (2004). Planotetraspora silvatica sp. nov. and emended description of the genus Planotetraspora. Int J Syst Evol Microbiol 54, 2053-2056.

Tamura, T., Suzuki, S. \& Hatano, K. (2000). Acrocarpospora gen. nov., a new genus of the order Actinomycetales. Int J Syst Evol Microbiol 50, 1163-1171.

Tamura, K., Dudley, J., Nei, M. \& Kumar, S. (2007). MEGA4: molecular evolutionary genetics analysis (MEGA) software version 4.0. Mol Biol Evol 24, 1596-1599.

Thompson, J. D., Gibson, T. J., Plewniak, F., Jeanmougin, F. \& Higgins, D. G. (1997). The CLUSTAL_X windows interface: flexible strategies for multiple sequence alignment aided by quality analysis tools. Nucleic Acids Res 25, 4876-4882.

Waksman, S. A. (1961). The Actinomycetes, vol. 2. Baltimore: The Williams \& Wilkins Co.

Yuan, L.-J., Zhang, Y.-Q., Guan, Y., Wei, Y.-Z., Li, Q.-P., Yu, L.-Y., Li, W.-J. \& Zhang, Y.-O. (2008). Saccharopolyspora antimicrobica sp. nov., an actinomycete from soil. Int J Syst Evol Microbiol 58, 1180-1185.

Zhou, Z.-H., Liu, Z.-H., Qian, Y.-D., Kim, S. B. \& Goodfellow, M. (1998). Saccharopolyspora spinosporotrichia sp. nov., a novel actinomycete from soil. Int J Syst Bacteriol 48, 53-58. 